\title{
Clinical risk management: an emerging agenda for psychiatry
}

\author{
David Roy
}

Health professionals are increasingly expected to take a share in the management of risk in a systematic manner. At its most blunt, clinical risk management is defined as the identification and planned response to negative events that occur during the provision of clinical care and can lead to litigation, the cost of this to the NHS is rapidly rising (Vincent \& Clements, 1995). There are, of course, much broader risks to any healthcare organisation, and the approach to risk management in any organisation needs to be systematic. At the start of the process the development of a list of clinical and non-clinical areas in which failures might occur can be overwhelming to clinicians and support staff alike. Rigorous prioritisation of the process is essential, and for practitioners the first rule of sound clinical risk management is common sense.

The individual clinician is well advised to consider the personal risks of clinical practice in the same way as one would ensure adequate insurance and pension arrangements for one's home and family. Medical records, including good note-keeping and communication, are the key to effective risk management. Issues relating to the general attitude and approach of clinical staff, particularly doctors, towards patients and carers is a growth area for complaints and dissatisfaction.

\section{Complaints procedures}

There is a growing volume of complaints within the NHS. Although related to the notion of patient rights and expectations fostered by charter standards as part of government policy, there is also a brief within trusts that complaints are an important means of empowering patients and ensuring the individual's voice is heard.

Trusts are now required to have an elaborate complaints system (DoH, 1995). The method of investigation and outcomes are closely monitored by purchasers. If the complaint relates to clinical decision making, the attitude of the doctor (a common complaint) or the quality of communications between professionals, and moves beyond the first stage, or is particularly serious, consultation with a legal advisor is recommended.

Occasionally the complaints department will pick up a run of complaints relating to a particular clinician, and the medical director might be asked to investigate, and on occasion, clinical peer review instituted in a particular area. This might result in more formal action. Although complaints may lead to disciplinary action against a clinician, formal proceedings are still rare.

\section{Serious incident inquiries}

Serious incidents relate to, for example, personal injury following assault with a weapon, inpatient suicide, homicide by a patient, or violence placing other patients significantly at risk. Many trusts regularly investigate outpatient suicides as well. Health Authorities have a system for the notification of most serious incidents which is managed upwards to the NHS Executive and Department of Health. The Ministerial Office has an, not surprising, aversion to hearing about such incidents in the press without warning. Depending on local policy, serious incidents may result in a Board Level Inquiry. often with an external person invited to join the panel. Such inquiries are costly, time consuming, and always come up with a large number of recommendations for a service.

If a homicide has occurred, the current situation requires there to be both Board Level Inquiry and then, often several years later, an Independent External Inquiry convened by the Health Authority, the outcome of which is published, and in which individual doctors are named and often criticised. These inquiries aim to find fault, and clinicians should not underestimate the damage to personal reputation and self-esteem that can result, particularly if the panel feels it has been poorly served by the clinicians concerned. One question that will be asked after a case has become public is whether the clinical staff have been subject to disciplinary procedures by their employers. Paradoxically, it should be easier to resist finger pointing when 
clinical risk management is high on the local agenda and these issues become key areas for attention by a local risk management committee.

The process of automatic independent inquiry is under review by the Department of Health. One alternative suggestion is for an early single inquiry to be held at local level with an external chair appointed in collaboration with the purchasers and the NHS Executive with the recommendations being made public once a local action plan has been agreed. This will go some way to reducing the negative impact on local services, lower costs and streamline the system significantly while still serving the public interest (Roy et al, 1996).

\section{The inquest}

The coroner's inquest is to establish the cause of death, not to punish. However, a coroner may comment in his court about the professional conduct of an individual if he feels it contributed to the death. This might have serious consequences for a doctor in terms of subsequent litigation, or damage to reputation through media coverage. When doctors are asked to appear at an inquest, usually as the result of a report that has been prepared, they should have already been in discussion with the local manager responsible for the associated investigation, and the senior clinician or clinical director. If there is any suggestion that the case might be contentious, independent legal advice on the report, and possible representation, should be sought. Trusts may have a legal representative present who will be wanting to protect the reputation of the trust, and therefore, the doctor. It is advisable to ensure the trust's support or independent advice if a jury inquest is proposed, although there will be some circumstances in which a jury inquest is always held, for example, with death on a railway property, where the psychiatrist might not even be called to the court.

\section{Litigation}

It is clearly not appropriate to allow the fear of litigation to set the tone of one's clinical practice. A sensitive approach to relationships with patients and relatives who have complaints goes a long way to smoothing the path. However, in some cases even the most conciliatory approaches may be fruitless. The correct clinical decision is not always accepted by the patient or relative, even after a second and third clinical opinion is sought to back this up. If litigation is being considered against a doctor, employing authorities may be less than supportive if the doctor is seen as difficult, and if local policy and procedure have not been followed. It is essential for all doctors to have independent medico-legal insurance no matter how supportive the employer. Trusts should advise doctors to seek additional independent advice if they are being asked to appear before an independent inquiry into a serious untoward incident. The individual doctor will then be well placed to receive appropriate support should litigation ensue, or the organisation wish to take disciplinary action against the doctor. For most disciplinary issues the British Medical Association will act on the doctor's behalf.

\section{The Care Programme Approach, Supervision Register and Supervised Discharge}

There is some case law around the responsibilities of Health and Local Authorities to provide satisfactory aftercare to a patient discharged under Section 117 of the Mental Health Act. The responsibility of individual consultant psychiatrists is less clear. There is a wide variation across providers in implementing the CPA and Supervision Register. As with any locally agreed policy, the consultant psychiatrist might be called to account if sensible policies have been flouted when care is being planned. If services deemed necessary by the consultant are not available in practice, for example a key worker cannot be allocated to a particularly vulnerable patient, it is important for this information to be clearly noted and drawn to the attention of the local managers if the patient is felt to be at risk as a result, and in particularly difficult cases, the letter copied to the Medical Defence Society. Increasingly consultants are asked to discharge patients home from the relative safety of an acute admission ward before they are ready to leave. Although these risks are taken on a daily basis, the consultant will still be held accountable for the ultimate decision taken to discharge.

Confidentiality is clearly an issue, particularly with the CPA and supervision register. The sharing of information between agencies is often essential, and sometimes in the public interest, but issues of confidentiality must be considered on a case by case basis. Care should also be taken when communicating with third parties in writing on behalf of a patient about their psychiatric history. A written waiver is advisable. Generally openness of communication and sharing of information with the patient is a good thing, although care should be taken not to do so with third party information without the consent of that party. Lipsedge (1995) emphasises the requirement on staff to obtain and give information, the obligation to convene care reviews when problems arise, and the putative benefits which might accrue to patients who are 
successfully established on the supervision register.

\section{Clinical guidelines}

There is a growth industry in the development of clinical guidelines taking evidence-based practice into account, but much of the work in developing these protocols is time-consuming and only indirectly related to the management of clinical risk. Issues of risk arise if guidelines are promoted by a national body, such as the Royal College of Psychiatrists' guidelines for the administration of ECT or rapid tranquillisation. Over time, if the guidelines find general acceptance with the profession and are increasingly adopted, those services or individuals who then do not practise accordingly place themselves at risk. Often, however, central guidelines are rather vague, encouraging services and groups of clinicians to agree local practice using the structure provided. Increasingly purchasers will compare notes and seek uniform protocols to be put in place, in the way that managed care is controlled by insurance companies in the United States. To avoid this situation the profession needs to take the lead, and adopt sensible and flexible protocols in a planned way. A Special Working Party on Clinical Assessment and Management of Risk (Royal College of Psychiatrists, 1996) has recently prepared a document on the assessment and clinical management of the risk of a psychiatric patient causing harm to another person. The assessment of risk to others will always be the most anxiety provoking of areas in clinical risk management.

\section{Induction, training and consultant responsibility}

Scant attention has been paid in the past to training medical students and psychiatrists in the practicalities of risk management. There is a need to raise the profile of risk management in training, and also for consultants and the organisation to ensure a full induction programme for all new doctors. Trusts are particularly vulnerable when dependent on short-term locums, particularly when reviewing the liability of the consultant on call. Recent inquiries have highlighted the potential for poor decision making when locums are unfamiliar with the policies and procedures of the trust, and the responsibility of the out of hours on-call hierarchy is important in this area. Measures such as checklists and handbooks are a good example of simple risk management procedure. The development of strategy is a growth area in the health service, with clinical risk management strategies for all healthcare organisations a rapidly emerging priority. Increasingly trusts are looking to clinicians to take an active role in the development of local risk policies, and in the very early stages of the development of this process, with clinical autonomy and the role of the consultant under scrutiny, it is surely in our interest to take a lead in this process, onerous though it may seem.

\section{References}

DEPARTMENT OF HEALTH (1995) Acting on Complaints: Revised Policy and Proposals for the new NHS Complaints Procedure in England. March 1995 (EU(95)37). London: HMSO.

LIPSEDGE. M. (1995) Clinical risk management in psychiatry. In Clinical Risk Management (Ed. C. Vincent). pp. 276293. London: BMJ publishing.

Royal College of Psychiatrists (1996) Assessment and Clinical Management of Risk of Harm to other People. Special Working Party on Clinical Assessment and Management of Risk. Council Report CR 53. London: RCPsych.

RoY, D., LeLuor, P. \& Gurre, H. (1996) Inner City Mental Health. A report to the Council of the NHS Trust Federation. NHS Trust Federation.

VinCENT, C. \& CLEMENTS, R. (1995) Clinical risk management - why do we need it? Clinical Risk, 1, 1-4.

David Roy, Medical Director, Lambeth Healthcare NHS Trust, 108 Landor Road, London SW9 9NT 